\section{Bezoares gastrointestinales: mitos y realidades}

\author{
RICARDO ESPINOZA GONZÁLEZ
}

\section{Gastrointestinal bezoars}

Gastrointestinal bezoars are a concretion of indigested material that can be found in the gastrointestinal tract of humans and some animals. This material forms an intraluminal mass, more commonly located in the stomach. During a large period of history animal bezoars were considered antidotes to poisons and diseases. We report a historical overview since bezoars stones were thought to have medicinal properties. This magic conception was introduced in South America by Spanish conquerors. In Chile, bezoars are commonly found in a camelid named guanaco (Lama guanicoe). People at Central Chile and the Patagonia believed that bezoar stones had magical properties and they were traded at very high prices. In Santiago, during the eighteenth century the Jesuit apothecary sold preparations of bezoar stones. The human bezoars may be formed by non-digestible material like cellulose (phytobezoar), hair (trichobezoar), conglomerations of medications or his vehicles (pharmacobezoar or medication bezoar), milk and mucus component (lactobezoar) or other varieties of substances. This condition may be asymptomatic or can produce abdominal pain, ulceration, gastrointestinal bleeding, gastric outlet obstruction, perforation and mechanical intestinal obstruction. We report their classification, diagnostic modalities and treatment.

(Rev Med Chile 2016; 144: 1073-1077)

Key words: Bezoars; Gastric Outlet Obstruction; History of Medicine; Intestinal Obstruction.
Facultad de Medicina y Clínica Universidad de los Andes. Santiago, Chile.

Recibido el 18 abril de 2016, Aceptado el 6 julio de 2016.

Correspondencia a: Dr- Ricardo Espinoza G. Facultad de Medicina y Clínica Universidad de los Andes Santiago, Chile. respinoza@clinicauandes.cl
$\mathrm{E}$ 1 bezoar consiste en una concreción calculosa que puede encontrarse en el tubo digestivo de las personas y algunos animales, en especial mamíferos rumiantes. Corresponde a la acumulación de un material que no se digiere, conformando una masa intraluminal que puede llegar a producir variadas manifestaciones clínicas ${ }^{1}$.

El conocimiento de la existencia de los bezoares animales data de tiempos inmemoriales y también se arrastra desde épocas muy pretéritas, primero el asombro y luego la asociación de éstos con propiedades particulares, como protectores de la salud ${ }^{2,3}$. Para algunos, el término bezoar proviene de dos palabras hebreas, bel, señor y zaar, veneno, es decir, señor de los venenos o contraveneno ${ }^{1-3}$. Sin embargo, el conocimiento más cercano de los bezoares proviene de la medicina oriental, y ello coincide con que la palabra bezoar deriva más probablemente del vocablo árabe badzher o el panzehr del Persa y que en ambos casos significa antídoto, es decir, tendría propiedades protectoras, principalmente contra envenenamientos, aunque también tendría efecto sobre el estado de ánimo de quienes lo utilizaban $^{4,5}$. De hecho, debemos al sevillano árabe Ibn Zuhr, para nosotros Avenzoar, la primera descripción de las propiedades de los bezoares, una de las razones por las que este médico arábico medieval logró fama como clínico y terapeuta. Fue esta medicina arábica la que actuó primero como receptora y posteriormente desarrolló el legado de los griegos, la que creó las bases de la medicina occidental a partir de los siglos X y XI; y con ello se introdujo en Europa el conocimiento y aprecio por los bezoares ${ }^{6,7}$. De hecho, y como veremos, los bezoares fueron atesorados por reyes 
y plebeyos, han sido incluidos en la literatura y formaron parte de nuestra cultura hasta muy recientemente ${ }^{8}$.

\section{Algunos hechos históricos de interés}

Como insinuamos, los bezoares de animales fueron altamente cotizados y valorados en la Europa medieval, por un lado por la creencia en sus propiedades como antídoto y también por la belleza de algunos de ellos. En este ambiente de superstición, llegaron a ser tan valorados como las piedras preciosas, y sabemos que la reina Isabel I de Inglaterra poseía uno particularmente bello, que decoraba su corona ${ }^{8}$. Asimismo, sabemos que el emperador Carlos V de España también hacía uso de los bezoares, especialmente en una época en la que los envenenamientos en la corte eran bastante frecuentes. Ya dijimos que médicos árabes como Rhazes y Avenzoar recomendaban con fuerza los bezoares y "Arnaldo de Vilanova, español y uno de los más grandes médicos de la Edad Media, del siglo XIII, y su discípulo Raimundo Lulio la preconizaron (a la piedra bezoar) como el antídoto más eficaz, de modo tal que cuando los españoles llegaron a América la traían para defenderse de las mordeduras de animales venenosos y si tuvieron alguna inquietud de que escaseara la droga pronto lograron disiparla, pues encontraron que otros rumiantes del Nuevo Mundo también la producían", nos ha dicho Laval9.

En el año 1256, Alfonso X el Sabio había mandado a traducir al castellano el Libro de Picatrix, obra de origen árabe de alrededor del año 1000, que era un verdadero tratado de magia, y que entre sus muchas indicaciones, señalaba cómo preparar diversos talismanes en base a la utilización de las piedras bezoares extraídas de animales. Nicolás Monardes, médico de Sevilla, en el año 1574 en su "Historia medicinal de las cosas que se traen de nuestras Indias occidentales que sirven en medicina", se explayaba sobre las virtudes de las piedras bezoares, explicando "en tomando peso de tres gramos de esta piedra con agua de lengua de buey, han fácilmente sanados"9. Poco tiempo después el jesuita José de Acosta en su "Historia Natural y Moral de las Indias", publicada en Sevilla en 1590, señalaba: "El efecto principal de la piedra bezoar es contra venenos y enfermedades venenosas... se aplica molida y echada en algún licor que sea a propósito del mal que se cura. Unos la toman en vino, otros en vinagre, en agua de azahar, de lengua de buey, de borraja y de otras maneras, lo cual dirán los médicos y boticarios. No tiene sabor alguno la piedra bezoar..." Agrega que a la ya conocida acción de las piedras bezoares "en caso de intoxicación, cualquiera sea el veneno causante" también sirven para "la melancolía y el mal de corazón y calenturas pestiferas".

En Francia, Carlos IX poseía un valioso bezoar. Pues bien, uno de los cocineros del rey, acusado de haber robado su cuchillería de plata fue condenado a muerte; en ese momento intervino el médico de la corte, Ambrosio Paré. Este encontró propicia la ocasión para demostrar la inutilidad médica de los bezoares y destruir el mito; pidió cambiar la horca por el envenenamiento, para que, una vez ingerido el veneno, se le administrara al condenado una piedra bezoar que contrarrestara los efectos tóxicos, y si sobrevivía recuperaba la libertad. El rey accedió, pero, irremediablemente y transcurridas siete horas, el cocinero murió en medio de intensos dolores; con ello el médico daba prueba de la fraudulenta acción de los bezoares como antídoto. Pero se cuenta que el rey no modificó su fe en los bezoares, pensó que había sido engañado al adquirir uno falso y de inmediato ordenó comprar otro ${ }^{8}$. Laval ${ }^{9}$ nos dice así: "No obstante esta prueba tan demostrativa la piedra bezoar continuó gozando del favor ilimitado de médicos y pacientes".

Concluida la Edad Media, el naturalista francés Georges Louis Leclerc, más conocido como conde de Buffon (1707-1788), recopiló buena parte del conocimiento sobre los animales productores de bezoares, y en su "Historia Natural, general y particular", señalaba: "Las bezoares en que se han hallado o supuesto más virtudes y propiedades, son las bezoares orientales, las cuales, como hemos dicho, provienen de las cabras, de las gazelas y de los carneros que habitan en las altas montañas del Asia. Las bezoares de inferior calidad, llamadas occidentales, provienen de las llamas o alpacas, que no se encuentran sino en las montañas de la América meridional. Finalmente, las cabras y lasgazelas de Africa producen también bezoares, aunque no tan buenas como las Asia"10.

Buffon añade: "Los viageros aseguran que los grandes ximios de las partes meridionales del Asia producen piedras bezoares que se encuentran en su estómago, y cuyas qualidades son superiores a las de las bezoares de las cabras y de las gazelas. Estos grandes ximios de las partes meridionales de la India 
son el vanderú y el duco"11. En otro tomo Buffon comenta: "Podemos asegurar que la mayor parte de los cuadrúpedos, a escepción de los carniceros, dan bezoares, y que también se encuentran en los cocodrilos y en las culebras grandes" 12 .

Transcurrieron muchos años hasta que la creencia en las propiedades atribuidas a los bezoares comenzara gradualmente a declinar en Europa, especialmente desde el siglo XVIII en adelante. En cambio, a las Indias recientemente descubiertas, y en etapa de colonización, se transfirió la creencia en los poderes mágicos de los bezoares, como desarrollaremos a continuación. No obstante, y hasta la actualidad, han sido numerosas las obras de literatura que han recurrido a los bezoares como un recurso mágico o curativo. Podemos mencionar, entre otros, la clásica novela de Oscar Wilde, "El retrato de Dorian Grey"; más recientemente, en la serie de Harry Potter también se hace referencia a los poderes de los bezoares.

En ningún caso, sin embargo, queda claro qué motivó a lo largo de la historia la creencia en las propiedades de los bezoares. Sabemos lo apreciada que fue la botánica desde los tiempos de Hipócrates en toda la terapéutica. Y puesto que, como decía Buffon: "Se puede deducir que en general las bezoares no son más que un residuo del nutrimento vegetal... se engendra sino en los (animales) que se alimentan de plantas: que siendo las yerbas más vigorosas y activas en las montañas del Asia Meridional que en ningún otros país del mundo, las bezoares, que son sus residuos, tienen también más virtud que las otras"13. Podemos plantear como hipótesis que, identificadas ciertas hierbas como medicinales, se pensaba que un bezoar las concentraba de manera insuperable.

\section{Los bezoares en nuestra cultura chilena}

Hemos comentado que los españoles transmitieron a estas tierras la creencia en los poderes de los bezoares, de modo que, efectivamente, había preocupación por ellos y formaron parte de las descripciones de los muchos hallazgos en los animales de este hemisferio. Se han clasificado los bezoares en la "variedad oriental que proviene principalmente de la Capra aegagrus, una cabra que vive en estado salvaje en las montañas de Persia y el Cáucaso", hoy Turquía, Irán y Afganistán y el bezoar occidental o americano que procede de diversas especies animales: llamas, vicuñas, guanacos, alpacas, tapires y venados americanos". Sigue Laval: "El bezoar que se encuentra en Chile proviene del guanaco". El Padre Diego Rosales en su "Historia General del Reyno de Chile" expresa que "crían muchos en el vientre piedras vezares que fraguan de yerbas muy cordiales y expulsivas de todo veneno. Y sobre un palito se va formando la piedra, adquiriendo unas capas sobre otras con que toma cuerpo y grandeza". Nos dice también Laval que el Padre Miguel de Olivares en su "Historia Militar, Civil y Sagrada de los acaecido en la conquista de Chile", dejó escrito: "El guanaco animal montaraz...cría en el vientre una particular bolsita y en ella una o muchas piedras besuares que tienen no vulgar crédito entre los simples de la medicina". El mismo Miguel de Olivares, cuando describe las costumbres de los indios en "Los jesuitas en la Patagonia: Las Misiones en la Araucanía y el Nahuelhuapi" (1593-1736) señala: "De los guanacos también cojen la carne para su alimento, $i$ de ellos sacan las piedras bezares, que se estiman como es notorio". En las notas señala: "Se comprende que un medicamento al cual la ignorancia i la superstición atribuian tan maravillosas cualidades, debia ser mui buscado i debía tener un precio exorbitante" ${ }^{14}$.

En las notas que dejó el insigne historiador Diego Barros Arana ${ }^{15}$ en su "Historia General de Chile", señala: "Los indios tenían muchas casas provistas de aves domésticas, pero lo que habia de más notable era una especie de oveja que los habitantes llaman carnero de la tierra (el guanaco). Nosotros matamos cuarenta y tres; y yo encontré en el estómago de uno trece piedras de bezoar (o bezar) de diferentes figuras. Aunque todas eran verdes cuando las saqué del estómago, con el tiempo tomaron color de ceniza..." Añade que "Desde que se comenzaron a apreciar estas piedras, dicen que los indios han hecho algunas artificiales y adulteradas" ${ }^{15}$. El mismo historiador comenta que "el comercio de estas piedras tomó en el Perú y en Chile desde esos años una notable importancia, lo que explica estas falsificaciones". Barros Arana nos cuenta que, la ignorancia y la superstición también atribuían a los bezoares otras virtudes, como que "el que llevaba al cuello una piedra de esta especie no podía dejar de ser feliz" $y$, dado los precios exorbitantes en que se llegaban a transar, "los pobres, que no tenían cómo comprar un talismán de esta naturaleza, lo tomaban alquilado en ciertos días" 15 .

Las piedras bezoares, en Chile, eran apreciadas 
y fueron, sin duda, muy solicitadas. En la Botica de los Jesuitas de Santiago, magistral botica que aportó notablemente a la medicina chilena hasta 1767 , los religiosos alemanes, a la vez que farmacéuticos, contaban con preparados de piedra bezoar, en base a carbonato de cal o creta precipitada que se administraba al interior en algún vehículo líquido, como lo señalaba el padre de Acosta, pero "también al exterior: pulverizándola dentro de una incisión practicada en el punto preciso en que el enfermo había sido mordido"'.

La creciente educación de la población y el desarrollo científico han relegado los usos medicinales de la piedra bezoar de los animales a los relatos históricos y fantasías.

\section{Los bezoares en humanos}

La medicina actual reconoce que los bezoares en humanos, aun cuando constituyen casos poco frecuentes, deben ser correctamente diagnosticados y tratados. Hoy se clasifica a los bezoares en cinco tipos, dependiendo de su principal composición. En primer lugar están los fitobezoares, que dan cuenta casi de la mitad de todos los bezoares y habitualmente se asocian a alimentos que contienen altas cantidades de celulosa. Un especial tipo de fitobezoar es el causado por la ingesta excesiva de caquis, muy difícil de disolver o fragmentar. Le siguen los tricobezoares, compuestos fundamentalmente por pelos y restos alimentarios ${ }^{1,4,16}$. Los tricobezoares, con frecuencia, se asocian a patología psiquiátrica y a tricofagia. Una condición particularmente poco frecuente y compleja la constituye el tricobezoar que se inicia en el estómago pero se prolonga por una extensión variable en el intestino, pudiendo llegar hasta el colon, condición que se ha identificado como síndrome de Rapunzel ${ }^{17-19}$. Los otros son los fármacobezoares, los lactobezoares y los polibezoares. Los fármacobezoares o bezoares ocasionados por medicamentos se han descritos en asociación con antiácidos, ciertos laxantes, la colestiramina y el sucralfato, entre muchos otros. En su patogénesis se han considerado los medicamentos que alteran la motilidad gastrointestinal, como los opioides y bloqueadores neuromusculares, y los que disminuyen la acidez gástrica ${ }^{2,4}$. Los lactobezoares son secundarios al uso de fórmulas lácteas y se observan en recién nacidos e infantes prematuros ${ }^{1,3,16}$. Por último, los polibezoares pueden formarse de una amplia gama de materiales ingeridos, como metales y plásticos ${ }^{2,3,16}$.

La localización más frecuente de un bezoar es la gástrica, seguida por los bezoares del intestino. $\mathrm{La}$ incidencia es bastante menor a 0,5\% entre aquellos sujetos que son sometidos a una endoscopia digestiva alta ${ }^{16}$. Si bien un paciente con un bezoar puede permanecer asintomático por largo tiempo o sólo tener malestar abdominal vago, la presencia de un bezoar gastrointestinal puede ser causa de dolor abdominal, hemorragia, anorexia, vómitos, baja de peso, disfagia, masa palpable, diarrea o constipación, obstrucción intestinal y aun perforación del tubo digestivo ${ }^{1,3,4,16}$.

En la formación de un bezoar pueden influir condiciones particulares del paciente, como todas aquellas que alteren la anatomía o funcionalmente el vaciamiento gástrico ${ }^{1,3,4,16}$. Por lo mismo son más frecuentes de observar postgastrectomía ${ }^{3}$, con una incidencia de hasta 12\%. En la actualidad se han descrito bezoares como complicación de una banda gástrica como parte del tratamiento de pacientes obesos ${ }^{20}$.

El diagnóstico puede ser sospechado por los antecedentes clínicos y síntomas, pero el diagnóstico se hará prácticamente por medio de cualquier método de imágenes, sea esta una radiografía simple con o sin medio de contraste, la ultrasonografía, la tomografía axial ${ }^{1,3,16}$ e incluso por medio de resonancia magnética ${ }^{21}$, pero es la endoscopia digestiva el examen de elección. A su vez, la endoscopia proporciona, en muchos casos, la posibilidad de tratarlos, ya sea por medio de su fragmentación, como terapia única o en combinación con otros métodos. Esto, que es válido para los fitobezoares, es menos posible ante un tricobezoar, que generalmente va a requerir de tratamiento quirúrgico ${ }^{1,3,16}$.

La disolución química de ciertos bezoares se ha intentado con celulasa, acetilcisteína, papaína, bicarbonato de sodio y muchos otros produc$\operatorname{tos}^{1,3,4,16}$. La más novedosa indicación corresponde a la instilación de Coca-Cola ${ }^{\circledR}$ como lo señaló $\operatorname{Ladas}^{22}$ en el año 2002. Estudios más recientes ${ }^{23,24}$ dan cuenta del éxito de la Coca-Cola ${ }^{\circledR}$ en cualquiera de sus formas comerciales, en casi $50 \%$ de los pacientes, aun cuando no está completamente aclarado su mecanismo de acción y los volúmenes óptimos. La indicación más recomendada es la ingesta o instilación de $3.000 \mathrm{~mL}$ en $12 \mathrm{~h}$. La terapia combinada endoscópica y química tiene 
éxito en más de $90 \%$ de los bezoares gástricos y la cirugía queda reservada, en general, cuando ésta fracasa o existe alguna complicación. Por tanto, el tratamiento de un bezoar va a depender importantemente de la localización y su causa, además de la condición de presentación.

Finalmente, tenemos que recordar que el objetivo del tratamiento es remover el bezoar gastrointestinal y prevenir su recurrencia.

\section{Referencias}

1. Andrus $\mathrm{CH}$, Ponsky JL. Bezoars: Classification, Pathophysiology, and Treatment. Am J Gastroenterol 1988; 83: 476-8.

2. Taylor J, Streetman DS, Castle SS. Medication Bezoars: A Literature Review and Report of a Case. Ann Pharmacother 1998; 32: 940-6.

3. Dikicier E, Altintoprak F, Ozkan OV, Yagmurkaya O, Uzunoglu MY. Intestinal obstruction due to phytobezoars: An update. World J Clin Cases 2015; 3: 721-6.

4. Eng K, Kay M. Gastrointestinal Bezoars: History and Current Treatment Paradigms. Gastroenterol Hepatol 2012; 8: 776-8.

5. Williams RS. The fascinating history of bezoars. Med J Aust 1986; 145: 613-4.

6. Sergio de Tezanos Pinto S. Breve Historia de la Medicina Universal y notas sobre historia de la medicina chilena. Editorial Universitaria. Santiago, Chile. 1979.

7. P. Laín Entralgo. Historia de la medicina. Masson SA. Barcelona, España. 2001.

8. Howard W. Haggard. The Lame, The Halt, and The Blind. Harpers \& Brothers Publishers. New York, USA. 1932.

9. Enrique Laval M. Biblioteca de Historia de la Medicina en Chile. II. Botica de los jesuitas de Santiago. Stanley. Santiago, Chile. 1953.

10. Compendio de la Historia Natural de Buffon. Tomo VIII. Imprenta de Villalpando. Madrid. 1804.

11. Compendio de la Historia Natural de Buffon. Tomo V. Imprenta de Villalpando. Madrid. 1802.

12. Obras completas de Buffon. Impr. de Bergnes y $\mathrm{C}^{\mathrm{a}}$. Barcelona. 1833.
13. Historia Natural, general y particular, por el conde de Buffon. Tomo XIII. Imprenta de la Viuda de Ibarra, Madrid. MDCCLXXXXIV.

14. Jesuita Miguel de Olivares. Los jesuitas en la Patagonia. Las Misiones en la Araucanía y el Nahuelhuapi (15931736). Ediciones Continente. Buenos Aires, Argentina. 2011.

15. Diego Barros Arana. Historia General de Chile. Tomo V, Capítulo Vigésimoprimero. Editorial Universitaria. Santiago, Chile. 2000.

16. Iwamuro M, Okada $H$, Matsueda $K$, Inaba $\mathrm{T}$, Kusumoto $\mathrm{C}$, Imagawa A et al. Review of the diagnosis and management of gastrointestinal bezoars. World J Gastrointest Endosc 2015; 7: 336-45.

17. Vaughan ED, Sawyers JL, Scott HW. The Rapunzel syndrome. An unusual complication of intestinal bezoar. Surgery 1968; 63: 339-43.

18. Anzieta J, Felmer O, Gabrielli M, Venturelli F, Sánchez G, Torrijos C. Obstrucción intestinal causada por Tricobezoar: Síndrome de Rapunzel. Rev Med Chile 2008; 136: 1027-30.

19. Belsky J, Whitcomb V, Zimmerman E, Stankovic C. Rapunzel Syndrome. Diagnosis via Radiograph and History. Pediatr Emerg Care 2014; 30: 352-3.

20. Cortés C, Silva C. Bezoar gástrico como complicación de banda gástrica en manejo de obesidad mórbida. Caso clínico. Rev Med Chile 2008; 136: 1457-9.

21. Ben Cheikh A, Gorincour G, Dugougeat-Pilleul F, Dupuis S, Basset T, Pracros JP. Gastric trichobezoar in an adolescent presenting with anemia: ultrasound and MRI findings. J Radiol 2004; 854: 411-3.

22. Ladas SD, Triantafyllou K, Tzathas C, Tassios P, Rokkas T, Raptis SA. Gastric phytobezoar may be treated by nasogastric coca-cola lavage. Eur J Gastroenterol Hepatol 2002; 14: 801-3.

23. Lee BJ, Park JJ, Chun HJ, Kim JH, Yeon JE, Jeen YT, et al. How good is cola for dissolution of gastric phytobezoars? World J Gastroenterol 2009; 15: 2265-9.

24. Ladas SD, Kamberoglou D, Karamanolis G, Vlachogiannakos J, Zouboulis-Vafiadis I. Systematic review: Coca-Cola can effectively dissolve gastric phytobezoars as a first-line treatment. Aliment PharmacolTher 2013; 37: 169-73. 